\title{
CALCULATION OF FERROMAGNETIC STATE AND CRITICAL TEMPERATURE IN TRANSITION METAL DOPED III-V WURTZITE SEMICONDUCTORS
}

\author{
M. A. SHAMSUL, M. SHAHJAHAN AND M.M. RAHMAN*1 \\ Center for Advanced Research in Sciences, University of Dhaka, Dhaka-1000, Bangladesh \\ Department of Physics, University of Dhaka, Dhaka-1000, Bangladesh
}

\begin{abstract}
The magnetic properties and electronic states of the transition metal doped III-V wurtzite compounds $\left(A_{l-x} M_{x}\right) \mathrm{N}$ are calculated using Korringa-Kohn-Rostoker Green's function method combined with the coherent potential approximation, where $A=\mathrm{Al}, \mathrm{Ga}$ and $M=3 d$ transition metal atoms namely $\mathrm{V}, \mathrm{Cr}, \mathrm{Mn}, \mathrm{Fe}, \mathrm{Co}, \mathrm{Ni}$ and $x$ is the fractional concentration of $M$. The positive value of the energy difference between ferromagnetic (FM) state and disordered local magnetic moment (DLM) state per unit cell denotes the magnetic phase stability. The total energy difference $\left(\mathrm{E}_{\mathrm{DLM}^{-}}\right.$ $\left.\mathrm{E}_{\mathrm{FM}}\right)$ is used to estimate the Curie temperature $\left(\mathrm{T}_{\mathrm{C}}\right)$ within the mean-field approximation. The calculated $\mathrm{T}_{\mathrm{C}}$ of $\mathrm{V}$ and $\mathrm{Cr}$ doped nitrides increases rapidly at lower concentrations and is found to be above the room temperature in the concentration range of $x=0.05-0.20$. The FM behavior in Mn doped $\left(\mathrm{Al}_{1-x} \mathrm{Mn}_{x}\right) \mathrm{N}$ is suppressed at the concentration range of $x=0.01-0.10$. A clear phase transition from DLM to FM state occurs at concentrations $x>0.10$. The energy difference in $\mathrm{Fe}, \mathrm{Co}$ and Ni doped materials, results in lower values of the DLM states, where the super-exchange interaction dominates over the FM one. The FM materials exhibiting $\mathrm{T}_{\mathrm{C}}$ above room temperature have applications in the field of spintronics.
\end{abstract}

Keywords: KKR-Green's function, Coherent potential approximation, Disordered local magnetic moment (DLM), Ferromagnetic materials, Curie temperature

\section{INTRODUCTION}

Diluted magnetic semiconductors (DMS) have been a context of great interest for possible application in the field of spintronics as they have the intrinsic properties of both semiconductor and ferromagnetic materials. Spintronic devices use both the electrical charge and spin of electrons to improve the functionality of the devices. The DMS or the half metallic state (González, et at. 2011) is found when a semiconductor is doped with $3 d$ transition metals (TM), which leads to the interesting transport properties with carriers of the one type of spin forming a new class of DMS materials.

\footnotetext{
* Corresponding author: <mmizan@du.ac.bd>.

${ }^{1}$ Department of Physics, University of Dhaka, Dhaka-1000, Bangladesh.
} 
Current research in spintronics is focused in the study of magnetic behavior of new materials, for example, magnetic ordering of dopants in semiconductors, magnetic phase stability of alloys formed by semiconductors doped with TM atoms. For a material to exhibit magnetic properties, there must be some form of ordination amongst the magnetic moments. For an antiferromagnet, the moments are aligned anti-parallel throughout the material, whilst for a ferromagnet the moments are parallel to each other. An important parameter for this ordering is temperature. If the temperature exceeds the ordering energy then the material will lose its magnetic properties. For ferromagnets, this temperature is called the Curie temperature. The carrier induced magnetic properties of DMS has demonstrated that the Curie temperature $\left(\mathrm{T}_{\mathrm{C}}\right)$ can be controlled by changing the carrier density (Ohno, 1992). Therefore, to synthesize a material with $\mathrm{T}_{\mathrm{C}}$ higher than room temperature (RT) has become one of the important topics in spintronics.

Early studies of DMS were focused on II-VI semiconductors, such as CdS, CdSe, ZnTe, $\mathrm{ZnS}$ and so on. Since many TM atoms adopt divalent ionic states, the TM impurities substitute with the divalent cations easily. However, most II-VI DMS are antiferromagnetic, or have low $\mathrm{T}_{\mathrm{C}}$ (Chambers, 2009). For this reason they are quite unattractive for application. Several attempts have been made to search DMS with their half metallic ferromagnetic and antiferromagnetic natures exhibiting perfect spin polarization at the Fermi level (Furdyana 1988, Bhatt et al. 2002, Sato et al. 2003, Bergqvist et al. 2007). Interest in the family of carrier induced III-V type DMS started with the discovery of ferromagnetism in 5\% Mn-doped InAs and GaAs with a Curie point of $110 \mathrm{~K}$ (Ohno et al. 2000). Dietl et al. (2002) tried to explain the carrier-controlled ferromagnetism in TM doped III-V semiconductors using the doubleexchange Zener model (Zener 1951) of ferromagnetism and enumerated how the doping of Mn ions initiates ferromagnetic behavior via hole-mediated exchange interaction. Firstprinciples calculation (Schilfgaarde et al. 2001) have been carried out to understand the underlying mechanism of carrier-induced ferromagnetism in TM doped III-V semiconductors. Based on Density Functional Theory there are mainly two approaches (a) the supercell approach in which the TM atom is doped in the substitutional cation site in a large supercell such that the impurities in two neighboring cells do not interact; (b) the coherent potential approximation (CPA) approach, which treats the DMS as a chemically disordered system. Recently, TM doped III-V oxides and nitrides are getting more interest because of the theoretical prediction and experimental realization of RT ferromagnetism (Beloufa et al. 2009, Coey et al. 2004, Han et al. 2016). Therefore, III-V type DMS opens up the new door of incorporating a variety of magnetic properties in the field of spintronics and is compatible with the present day electronics and the existing optical and electrical devices.

\section{FRAMEWORK OF CALCULATION}

We used Korringa-Kohn-Rostoker (KKR) Green's function method combined with the CPA for the calculation. First-principles calculations are used which is an efficient 
tool to describe magnetism as no parameters need to be introduced from experiments. The electronic structures of DMS are calculated based on the generalized gradient approximation (GGA) and muffin-tin potential approximation (MTA). GGA is a class of approximation to the exchange correlation (Akai, 1998) energy functional that depends upon the value of the electronic density and its gradient in space. MTA is used in this calculation, where the potential was assumed to be spherically symmetric inside the atomic sphere and constant in the interstitial regions. In DMS, TM impurities are introduced randomly into cation sites of the host semiconductor. This substitutional disorder is described by the CPA method (Shiba, 1971). We have calculated the electronic properties using CPA, where the averaged electronic properties were considered instead of individual properties for respective impurity configurations.

For the calculation of the magnetic phase stability, Curie temperature $T_{C}$ and the electron structures, the wurtzite semiconductors are systematically doped with $\mathrm{V}, \mathrm{Cr}, \mathrm{Mn}$, $\mathrm{Fe}$, Co and Ni TM atoms into the cation sites of host compounds upto 3, 5, 10, 15, 20 and 25 percent concentrations. Imaginary part at the Fermi level is approximately taken as $0.001 \mathrm{Ry}$ and the width of the energy contour is set as $1.5 \mathrm{Ry}$. The shape of the crystal potential is approximated by a muffin-tin potential, and the wave functions are calculated up to $l=2$, where $l$ is the angular momentum quantum number defined at each atomic site. Lattice parameters ' $a$ ' and 'c' represent the edge length of the basal plane hexagon and the axial height of the unit cell perpendicular to the basal plane, respectively. The $u$ parameter (internal parameter) describes the relative position of anion sub-lattice with respect to the cation sublattice along the ' $c$ ' axis in the wurtzite structure. The lattice constants of the wurtzite structure are shown in Table 1.

Table 1. Lattice constants and band-gap energies of wurtzite AIN and GaN are taken from (Juza et al. 1938).

\begin{tabular}{ccc}
\hline Compounds & GaN & AlN \\
\hline $\mathrm{a}=\mathrm{b}\left(\mathrm{A}^{0}\right)$ & 3.18 & 3.111 \\
$\mathrm{c}\left(\mathrm{A}^{0}\right)$ & 4.978 & 5.16 \\
$\mathrm{u}$ & 0.375 & 0.390 \\
$\mathrm{E}_{\mathrm{g}}(\mathrm{eV})$ & 3.4 & 6.015 \\
\hline
\end{tabular}

The total energy per unit cell of the wurtzite type structure is calculated for ferromagnetic (FM) state, where magnetic moments point in the same direction which is described as $\left(\mathrm{Al}_{1-\mathrm{x}} \mathrm{M}_{\mathrm{x} / 2}{ }^{1} \mathrm{M}_{\mathrm{x} / 2}{ }^{1}\right) \mathrm{N}$ and for disordered local moment (DLM) state with magnetic moments randomly oriented such that the net magnetization becomes zero $\left(\mathrm{Al}_{1-}\right.$ ${ }_{x} \mathrm{M}_{\mathrm{x} / 2}{ }^{1} \mathrm{M}_{\mathrm{x} / 2}{ }^{b} \mathrm{~N}$. In order to discuss the ferromagnetism of DMS, the total energies (TE) of both the ferromagnetic state and the DLM state are calculated and in particular the energy difference $\mathrm{E}=\mathrm{TE}(\mathrm{DLM})-\mathrm{TE}(\mathrm{FM})$ is estimated. However, lower energy state in FM 
phase compared to the corresponding DLM phase indicates the magnetic phase stability. Through numerical calculation we have found magnetic phase stability and RT ferromagnetism in $\mathrm{V}$ and $\mathrm{Cr}$ doped $\mathrm{AlN}$, which have been confirmed by various experimental works. Throughout the present calculations, we used the KKR-CPA package MACHIKANEYAMA 2002 developed by Akai (2002).

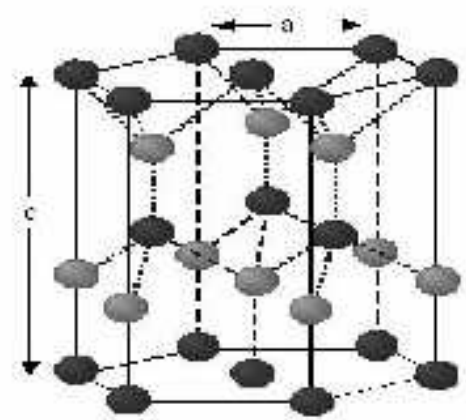

Fig.1. Unit cell of wurtzite structure (hcp)

\section{RESULTS AND DISCUSSION}

In this section, we present the calculated magnetic properties, Curie temperature $T_{C}$ and subsequently discuss the stability of magnetic phase and electronic structures in terms of density of states (DOS).

\section{Dilute Aluminum Nitride (AIN)}

In Table 2, magnetic moment, partial spin moment, energy difference $E$ as well as the Curie temperature, $\mathrm{T}_{\mathrm{C}}$ are tabulated for $10 \%$ concentration of TM atoms.

Table 2. Net magnetic moment (NM) per unit cell and local spin moment (SM) per atom in the FM state of $\left(\mathrm{Al}_{1-x} \mathrm{M}_{x}\right) \mathrm{N}$ and the energy difference between FM and DLM states for doped transition metal atoms.

\begin{tabular}{lllll}
\hline Materials & $\mathrm{NM}\left({ }_{\mathrm{B}} /\right.$ cell $)$ & $\mathrm{SM}\left({ }_{\mathrm{B}} /\right.$ atom $)$ & $\Delta \mathrm{E}(\mathrm{mRy})$ & $\mathrm{T}_{\mathrm{C}}(\mathrm{K})$ \\
\hline$\left(\mathrm{Al}_{0 \cdot 90} \mathrm{Ti}_{0 \cdot 10}\right) \mathrm{N}$ & 0.10 & 1.16 & 0.07 & 74 \\
$\left(\mathrm{Al}_{0.90} \mathrm{~V}_{0.10}\right) \mathrm{N}$ & 0.19 & 1.32 & 0.49 & 523 \\
$\left(\mathrm{Al}_{0 \cdot 90} \mathrm{Cr}_{0 \cdot 10}\right) \mathrm{N}$ & 0.29 & 2.19 & 0.47 & 493 \\
$\left(\mathrm{Al}_{0 \cdot 90} \mathrm{Mn}_{0 \cdot 10}\right) \mathrm{N}^{*}$ & 0.00 & 0.00 & 0.00 & - \\
$\left(\mathrm{Al}_{0 \cdot 90} \mathrm{Fe}_{0 \cdot 10}\right) \mathrm{N}$ & 0.49 & 3.29 & -0.70 & - \\
$\left(\mathrm{Al}_{0 \cdot 90} \mathrm{Co}_{0 \cdot 10}\right) \mathrm{N}$ & 0.21 & 1.39 & -0.17 & - \\
$\left(\mathrm{Al}_{0 \cdot 90} \mathrm{Ni}_{0 \cdot 10}\right) \mathrm{N}$ & 0.16 & 0.89 & -0.19 & - \\
\hline
\end{tabular}

* $\left(\mathrm{Al}_{1-x} \mathrm{Mn}_{x}\right) \mathrm{N}$ do not exhibit RT ferromagnetism for all $x$ (Sato et al. 2005).

The calculated results tabulated in Table 2 have been plotted in Fig. 2(a). The solid line is plotted for $10 \%$ concentrations of $\mathrm{TM}$ atoms. A positive energy difference 
indicates the stability of the FM state relative to the DLM state. From Fig. 2(a), it is observed that $\mathrm{Ti}, \mathrm{V}$, and $\mathrm{Cr}$ doped cases exhibit the stability of the FM state whereas for $\mathrm{Fe}, \mathrm{Co}, \mathrm{Ni}$, the lower value of DLM state dominates over the FM state. This indicates that $\mathrm{Fe}$, Co, Ni doped cases are unstable magnetic states. For lower concentration of $\mathrm{Mn}$ doped AlN, it does not show any ferromagnetic behavior while at higher concentration, at some cases it shows ferromagnetic behavior. According to some groups it has been reported that nitride semiconductors doped with $\mathrm{Mn}$ fabricated by reactive sputtering and molecular beam epitaxy shows ferromagnetic behavior, and the Curie temperature is above $300 \mathrm{~K}$ for Mn-AlN denoting the fact that it exhibit ferromagnetism above room temperature (Kanamori, 1974). Though some groups have reported that these Mn-doped nitride semiconductors are one of the candidate materials for devices operating above RT, on the other hand, other groups have reported that these materials do not exhibit ferromagnetism at RT (Song et al. 2005; Sato et al. 2005). Thus, whether these materials exhibit ferromagnetism above RT is yet to be justified. Hence, the clear evidence of ferromagnetism above RT in these materials is yet to be confirmed.
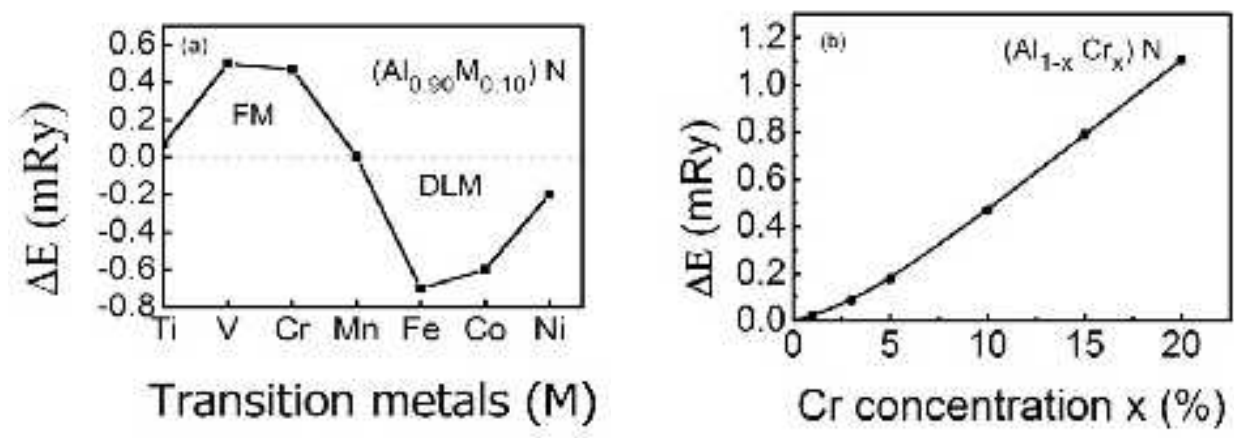

Fig. 2. (a) The energy difference ( $E$ ) per unit cell of $\left(\mathrm{Al}_{0.90} \mathrm{M}_{0.10}\right) \mathrm{N}$ between the $\mathrm{FM}$ and DLM states for a series of $3 d \mathrm{TM}$ atoms, (b) Energy difference $\mathrm{E}$ in mRy per unit cell for a range of $0 \%$ (nonmagnetic) to $20 \% \mathrm{Cr}$ in $\left(\mathrm{Al}_{1-\mathrm{x}} \mathrm{Cr}_{\mathrm{x}}\right) \mathrm{N}$.

Fig. 2 (b) shows the energy difference $E$ in mRy between FM states of $\left(\mathrm{Al}_{1-\mathrm{x}} \mathrm{Cr}_{\mathrm{x}}\right) \mathrm{N}$ and their respective DLM states as a function of $\mathrm{Cr}$ concentration. From the graph, we can conclude that energy difference for both the compounds gradually increases with the increasing concentrations of TM atoms. The curve is quite linear with increasing concentrations. Using the energy difference $\mathrm{E}$, we have also calculated the $\mathrm{T}_{\mathrm{C}}$ using the Heisenberg model in the mean field approximation, which can be expressed as $T_{C}=(2 / 3)$ ( $\mathrm{E} / \mathrm{xK}_{\mathrm{B}}$ ). We have calculated the $\mathrm{T}_{\mathrm{C}}$ for $\mathrm{Cr}$ and $\mathrm{V}$ doped cases and have discussed the results. The summarized results are plotted in Fig. 3. In Fig. 3(a) $T_{C}$ of $\left(\mathrm{Al}_{1-\mathrm{x}} \mathrm{Cr}_{\mathrm{X}}\right) \mathrm{N}$ is plotted against various concentration of Cr. The dotted lines represent the RT. From the figure we can infer that at lower concentrations of $\mathrm{Cr}$ atoms the values of $\mathrm{T}_{\mathrm{C}}$ increases sharply, however, at higher concentrations of the dopants $T_{C}$ provides stable value. The 
Heisenberg model in the MFA reports that the $\mathrm{T}_{C}$ of the DMS is proportional to $\mathrm{E}$. As a result, due to larger E between the DLM and FM states, the FM state for Cr doped case will be stabilized. It is evident from the graph that the $\mathrm{T}_{\mathrm{C}}$ is above RT starting from $3 \%$ $\mathrm{Cr}$ doped nitride. Thus, we can conclude that the $\mathrm{Cr}$ doped wurtzite AlN exhibits RT ferromagnetism. In the DMS with double exchange mechanism (Sato et al. 2003), $\mathrm{T}_{\mathrm{C}}$ is proportional to the square root of TM impurity concentration $\left(T_{C} \propto V_{x}\right)$. The plotted curve based on our calculation certainly follows the square root curve which indicates that our calculated results are consistent. In Fig. 3(b) the V doped case is quite similar to that of $\mathrm{Cr}$ doped case for concentration range of $(0 \%$ to $15 \%)$. But at higher concentration $(\mathrm{x}=0.17)$ of $\mathrm{V}$, the value of $\mathrm{T}_{\mathrm{C}}$ starts decreasing. This is because, the ferromagnetic super-exchange interaction mechanism is dominant at low concentrations of Vanadium, but the anti-ferromagnetic super-exchange interaction appears and reduces the stabilization of ferromagnetism at sufficiently high concentrations $(x>0.17)$. Consolidating the two cases, we can report that with a few constituent of $\mathrm{V}$ and $\mathrm{Cr}$ doped wurtzite AlN, the material exhibits RT ferromagnetism.
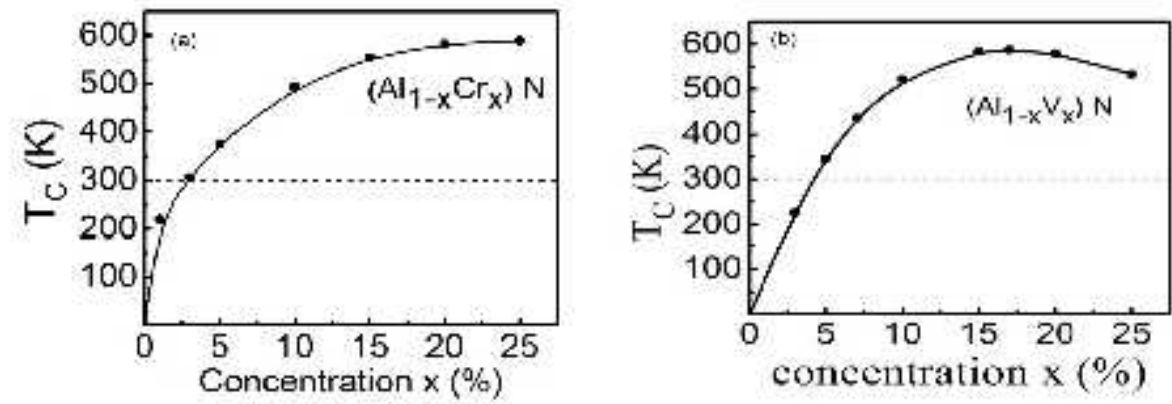

Fig. 3. (a) Curie temperature $\left(\mathrm{T}_{\mathrm{C}}\right)$ in $\mathrm{K}$ of (a) $\left(\mathrm{Al}_{1-\mathrm{x}} \mathrm{Cr}_{\mathrm{x}}\right) \mathrm{N}$, and (b) $\left(\mathrm{Al}_{1-\mathrm{x}} \mathrm{V}_{\mathrm{x}}\right) \mathrm{N}$ for a range of impurity concentrations from $0 \%$ (nonmagnetic) to $25 \%$ of magnetic atoms.

In order to fully understand the mechanism by which the FM state in Cr doped AlN compound is stabilized and at the same time to identify the contribution of $\mathrm{Cr}$ atom to the ferromagnetism, the total density of states (TDOS) and partial density of states (PDOS) for the $\left(\mathrm{Al}_{0.90} \mathrm{Cr}_{0.10}\right) \mathrm{N}$ compound were calculated. The positive sign in the vertical axis represents spin-up DOS, while the negative sign in the vertical axis denotes the spindown DOS. Figure 4(a) shows the total DOS of the undoped AIN semiconductor. From the Fig. 4(a) we can see that the Fermi region is located in the gap region, which indicates the semiconducting nature of AIN. The symmetric nature of spin-up and spin-down DOS curves confirms the nonmagnetic nature of AlN. Fig. 4(b) shows the TDOS and PDOS of $3 \mathrm{~d}$ states of $\mathrm{Cr}$ atom. As $\mathrm{AlN}$ is doped with $\mathrm{Cr}$ dopant, the d-states appear near the Fermi level. The d-states can be defined as three types of states, bonding which lies in valence band, non-bonding and anti-bonding states which are located above the valence band. 

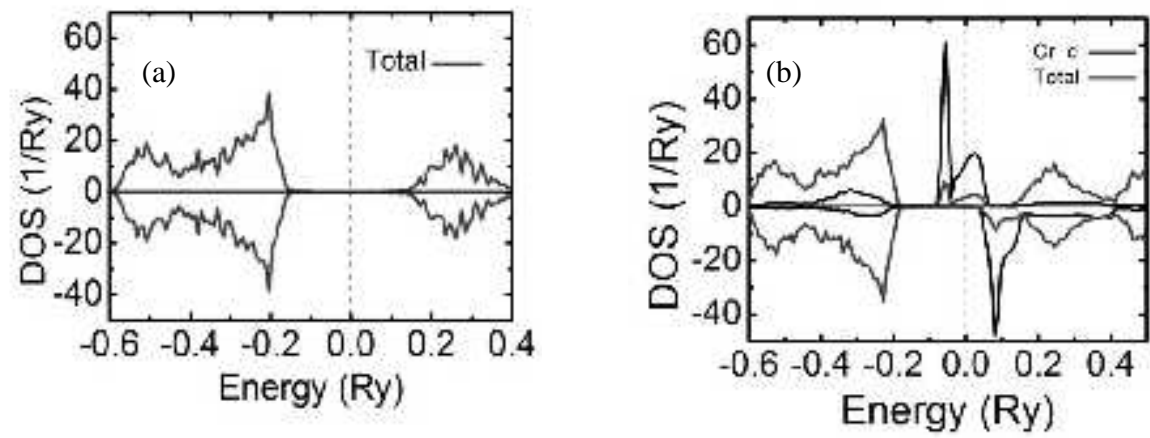

Fig. 4(a): Total DOS of host AlN semiconductor, and (b) total DOS and partial DOS of Cr doped AlN semiconductor

The TDOS curve confirms that due to the $\mathrm{Cr}$ substitution at the $\mathrm{Al}$ site, the compound has a ferromagnetic behavior. This behavior occurs because in the valence band near the Fermi level, majority of the spins are metallic and the minority spins are semiconducting. The non-bonding states are fully occupied and are located below the Fermi level but closer to the valence band. The anti-bonding states are split and we obtain localized and non-overlapping spin up and spin down states. The spin-up states are partially occupied. The spin-down states are above $E_{\mathrm{F}}$ and are slightly mixed with conduction band. This is similar to behavior of the same impurities in gallium nitride and is a result of Zener double exchange mechanism.

\section{Dilute Gallium Nitride (GaN)}

In Fig. 5(a) $\mathrm{T}_{\mathrm{C}}$ of $\left(\mathrm{Ga}_{1-x} \mathrm{Cr}_{x}\right) \mathrm{N}$ is plotted against various concentration of $\mathrm{Cr}$. The dotted lines represent the RT. From the figure, we can infer that for lower concentrations of $\mathrm{Cr}$ atoms the values of $\mathrm{T}_{\mathrm{C}}$ increases sharply, however, at the higher concentrations of the dopants $\mathrm{T}_{\mathrm{C}}$ provides stable values. This property is quite similar to that of $\mathrm{Cr}$ doped AlN case. Thus, we can conclude that the $\mathrm{Cr}$ doped wurtzite $\mathrm{GaN}$ exhibits room temperature ferromagnetism. Cr-doped $\mathrm{GaN}$ is expected to be one of the most stable candidates among all other transition metals doped GaN materials. (Sato et al. 2002)
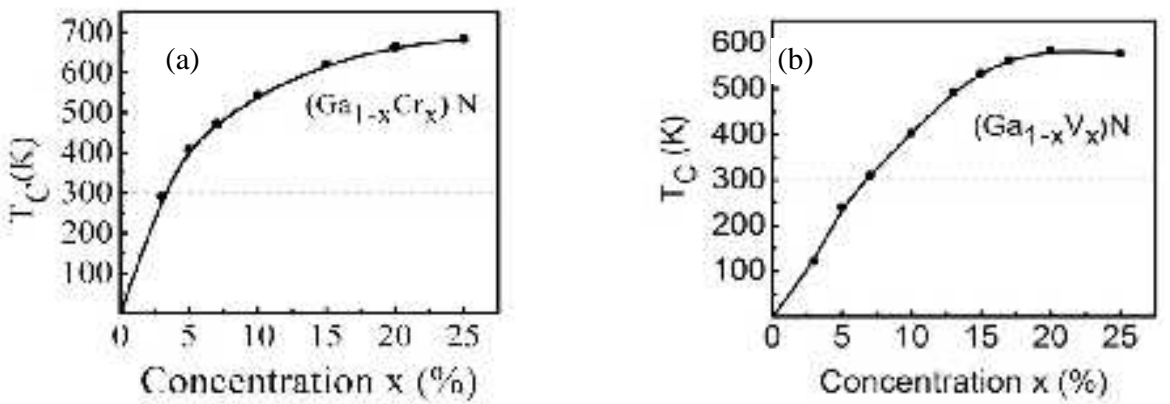

Fig. 5 Curie temperature $\left(\mathrm{T}_{\mathrm{C}}\right)$ in $\mathrm{K}$ of (a) $\left(\mathrm{Ga}_{1-x} \mathrm{Cr}_{x}\right) \mathrm{N}$, and (b) $\left(\mathrm{Ga}_{1-x} \mathrm{~V}_{x}\right) \mathrm{N}$ with respect to $\mathrm{Cr}$ and $\mathrm{V}$ concentrations, respectively. 
At low concentrations the Curie temperature increases rapidly but at higher concentrations the Curie temperature becomes steady. For $\left(\mathrm{Ga}_{1-x} \mathrm{~V}_{x}\right) \mathrm{N}$ and acceptor doped $\left(\mathrm{Ga}_{1-x} \mathrm{Cr}_{x}\right) \mathrm{N}$, ferromagnetic super-exchange interaction is responsible for the ferromagnetism, on the other hand, the ferromagnetic double-exchange interaction is dominant for high- $\mathrm{T}_{\mathrm{C}}$ in $\left(\mathrm{Ga}_{1-x} \mathrm{~V}_{x}\right) \mathrm{N}$, which is consistent with the results reported in the reference (Sato et al. 2003). Though the anti-ferromagnetic super-exchange interaction was dominant for high concentration $\mathrm{V}$ doped AlN in Fig. 3(b), but for high concentration of $\mathrm{V}$ doped $\mathrm{GaN}$ (Fig. 5(b), the anti-ferromagnetic super exchange is not dominant and hence the ferromagnetism remains steady. The underlying reason for this behavior is that the lattice constants of $\mathrm{AlN}$ are smaller than that of $\mathrm{GaN}$, and the effect of anti- ferromagnetic super-exchange interaction in $\left(\mathrm{Al}_{1-x} \mathrm{~V}_{x}\right) \mathrm{N}$ is strongest and easier to subdue the ferromagnetic interaction.

\section{CONCLUSION}

The structural, electronic, and magnetic properties of TM doped III-V wurtzite compounds are reported in this article using the CPA and GGA approximations. AIN and GaN-based DMS have very similar chemical trends in their magnetic stability and Curie temperature. Doping of $\mathrm{V}$ and $\mathrm{Cr}$ cases exhibit the stability of the FM state, whereas for $\mathrm{Fe}$, Co, and Ni impurities, the lower energy of DLM state dominates over the FM state. $\mathrm{T}_{\mathrm{C}}$ increases at low concentrations of $\mathrm{V}$ and $\mathrm{Cr}$ doped compounds and reaches a steady state at higher concentrations. From the presented results, one can infer that $\mathrm{Cr}$ and $\mathrm{V}$ doped AlN are good candidates for high $\mathrm{T}_{\mathrm{C}}$ ferromagnets as they exhibit ferromagnetism above RT. In addition, the TDOS of $\left(\mathrm{Al}_{0.90} \mathrm{Cr}_{0.10}\right) \mathrm{N}$ exhibits half-metallic and ferromagnetic behavior, because in the valence band close to the Fermi level, the majority spins (spin-up) are metallic, and the minority spins (spin-down) are semiconducting. The calculated FM half metals can be promising candidates for the nextgeneration spintronic and opto-electronic applications.

\section{ACKNOWLEDGMENTS}

The authors thankfully acknowledge the supports from the Center for Advanced Research in Sciences (CARS), University of Dhaka for providing advanced computing facilities.

\section{REFERENCES}

Akai, H. 1998. Phys. Rev. Lett. 81: 3002-3004.

Akai, H. 2002, http://kkp.phys.sci.osaka-u.as.jp.

Akai, H. and P. H. Dederichs. 1993. Phys. Rev. B. 47: 8739-8747.

Beheshtian, J., M. T. Baei, Z. Bagheri and A. A. Peyghan. 2012. Microelectronics J. 43(7): 452-455. 
Beloufa, A., Z. Bensaad, B. A. Soudini, N. Sekal, A. Bensaad and H. Abid. 2009. Int. J. Nano electronics Mater. 2(1) : 11-22.

Bergqvist. L. and P. H. Dederichs. 2007. J. Phys. Condens. Matter 19: 216220-216233.

Bhatt, R. N., M. Berciu, M. P. kennett and X. Wan. 2002. J. Supercond. 15: 71-83.

Chambers, S. A. 2009. Adv. Mat.21: 1-30.

Coey, J. M. D., A. P. Douvalis, C. B. Fitzgerald and M. Venkatesan. 2004. Appl. Phys. Lett. 84: 1332-1334.

Cui, X. Y., B. Delley, A. J. Freeman and C. Stampfl. 2006. Phys. Rev. Lett. 97: 016402.

Dietl, T., H. Ohno, F. Matsukura, J. Civert and D. Ferrand. 2000. Science.287: 1019-1022.

Endo, Y., T. Sato, A. Takita, Y. Kawamura and M. Yamamoto. 2005. IEEE Trans. Magn. 41: 2718-2720.

Furdyna, J. K. 1988. J. Appl. Phys. 64: R29-R64.

González, A., W. López and R. González. 2011. Solid State Commun. 151: 1794-1796.

Han. S. J., T. H. Jang, Y. B. Kim, B. G. Park, J. H. Park and Y. H. Jeong. 2016. Appl. Phys. Lett. 85: 2589-2592.

Juza, R. and H. Hahn. 1938. Z. Anorg. Allgem. Chem. 32: 66-68.

Kanamori, J. 1974. Journal de Physique Colloques, 35: 131-133.

Ohno, H. 1998. Science 281: 951-956.

Ohno, H., H. Munekata, T. Penney, S.V. Molnár and L.L. Chang. 1992. Phys. Rev. Lett. 68: 26642667.

Ohno, Y., D.Young, B. Beschoten, F. Matsukura, H. Ohno, D. Awschalom. 1999. Nature 402: 790792.

Sato, K., P. H. Dederics and H. K. Yoshida. 2003. Europhys. Lett. 61: 403-408.

Schilfgaarde, M.V., O.N. Mryasov. 2001. Phys. Rev. B 63: 233205-233206.

Shahjahan, M., T. Oguchi. 2016. J. Phys. Chem. Solids 93: 157-162.

Shiba, H.1971. Prog. Theor. Phys. 46: 77-94.

Song, Y. Y., P. H. Quang, V. T. Pham, K. W. Lee, S. C. Yu. 2005. J. Magn. Mater. 290-291: 13751378.

Zener, C. 1951. Phys. Rev. 82: 403-407. 